\title{
ABC LD - A NEW TOOLKIT FOR RAPID LEARNING DESIGN
}

\author{
Clive P. L. Young, Nataša Perović, University College London, United Kingdom
}

\section{Abstract}

ABC Learning Design (ABC LD) is a high-energy, hands-on curriculum development workshop from University College London (UCL). In just 90 minutes teaching teams work together to create a visual "storyboard". The storyboard is made up of pre-printed cards representing the type and sequence of learning activities (both online and offline) required to meet the module or programme learning outcomes. All the resources have been released under Creative Commons licenses and are free to download, adapt and use.

ABC LD is now popular across European tertiary education and beyond. Participants have found the workshop-based "sprint" approach to be quick, engaging and productive. The original UCL or "base" ABC LD is built around a collaborative and intensive 90' workshop in which module teams work together to produce a paper-based storyboard describing the student journey.

Over the last two years UCL has led an Erasmus+ project to develop and evaluate the ABC LD method with 12 partners (https://abc-ld.org). We have focused on localisation to institutional contexts and have explored the important link between storyboard designs and the Virtual Learning Environment. The main output is a freely downloadable Toolkit of resources and guides, enabling any college or university to adapt and adopt the method.

Although developed to promote blended learning, during the COVID emergency, some institutions have now modified ABC LD to be facilitated remotely to support their need for a rapid transition to online learning. ABC LD is proving an effective method in this new format, too

\section{What is the ABC LD Toolkit?}

Since being launched in 2015, UCL's ABC learning design method (Young \& Perović, 2016) has been widely adopted across universities in Europe and beyond. It is also being used in training, secondary education and other education -related sectors. The core of ABC LD is a highly successful sprint workshop format that engages academic teams in programme and module (re)design. Rapid institutional transition towards online learning due the 
Covid 19 pandemic has, if anything, reinforced the value of ABC LD's sprint approach. The suspension of live group work has disallowed the original format but the community of $\mathrm{ABC}$ LD enthusiasts has responded with energy and imagination to produce online $\mathrm{ABC}$ LD approaches that capture the essence of the method.

The Toolkit (2020) is a series of seven guides with accompanying resources that was coproduced in 2020 as the main output of the Erasmus+ project "ABC to VLE". The project was led by UCL and brought together 12 other universities to further develop, localise and evaluate the ABC LD learning design method. The partners were; University College Absalon, University of Amsterdam, Dublin City University, University of Helsinki, KU Leuven, University of Milan, University of Oxford (associate partner), Sorbonne University, Tallinn University, Polytechnic University of Timişoara, VIVES University of Applied Sciences, University College London (lead), and SRCE University of Zagreb. Some were already using the method and others were keen to try it. The project was focused on institutional adoptions of $\mathrm{ABC} L \mathrm{D}$, user experiences and how the designs could link to the local technical environment, and particularly their virtual learning environment (VLE) such as Moodle, Canvas or Blackboard. The project was still underway when the pandemic struck, so we were able to respond to the crisis by developing extra material, with input from the wider community. The main part of the Toolkit focuses on the original or "base" format and its variants. We are confident that we will be able to facilitate live workshops again soon. In the meantime, the core principles of co-design by teaching teams, apply to online as much as face-to-face activities and we are excited by the possibilities of the new online adaptations.

\section{The seven Toolkit guides}

The guides (G1 to G7) are not intended to be read in sequence, as many users will already be familiar with ABC LD method. Some knowledge from earlier guides may be assumed, though, in later ones. The titles are as follows.

- $\quad \mathrm{G} 1$ - Introducing ABC learning design - an overview of the principles.

- $\mathrm{G} 2$ - Base ABC LD and translations - how the live workshops run.

- $\mathrm{G} 3$ - Localising ABC - integrating ABC LD in local initiatives and policies.

- $\mathrm{G} 4-\mathrm{ABC}$ LD and the VLE - moving from designs to technical implementation.

- $\quad$ G5 - Does ABC LD work? - the evaluation of ABC LD method.

- G6 - Online ABC LD (Covid versions) - different approaches to replacing the live workshops

- $\quad$ G7 - The future of ABC LD - building the community and sharing practice.

The seven short guides, presented as an online resource, provide a cohesive narrative thread linking the various components of ABC LD method. The real value in the Toolkit 
however is the resource collection; tools, examples, variations and evaluations produced during the project and via the wider ABC LD community. These resources are linked from the Guides to enable those interested in the method to "dive deeper" at any point into specific resources, examples and ideas.

\section{How the Toolkit is intended to be used}

The guides address five user scenarios.

- If you are new to ABC LD, we'd suggest you start with G1. This guide introduces the underpinning theory, derived from Professor Diana Laurillard's "Conversational Framework" (2002; 2012) and her concept of "learning types". Videos from the live workshop give an impression of its dynamics, and the recorded explanations by Dr Clive Young and Nataša Perović (the co-creators of the ABC LD method) expand on how this version works and why.

- If you are interested in running ABC LD workshop, G2 breaks down the workflow of the original UCL version and introduces some variations from the Erasmus+ project partners and others. Part of the ethos of ABC LD method is to encourage and enable localisation. All ABC LD resources are downloadable and released under Creative Commons Attribution-NonCommercial-ShareAlike 4.0 International (CC BY-NCSA 4.0) license. You can adapt them to your needs, providing you keep the original attribution (and ideally share with the community). G2 provides ideas on how to do this.

- If you want to run an online ABC LD workshop, G6 offers some alternatives from the project and the community. Again, developers have been generous in providing resources. It is still probably best to look at G2 to see what the intended outcomes are and you will want to look at G4 and the technology support model. Look for the ABC LD “tool wheel” (or "app wheel”) for an interesting way to link pedagogical approaches to the tools available.

- If you want to adopt ABC LD method in your institution then G3 will provide some inspiration, and if you need evidence (for your management) or convincing (for you), G5 and the evaluation of ABC LD provides an interesting and positive story.

- If you want to join the ABC LD community, and/or create a local network, then G7 lists some ideas we have used in the project and beyond.

\section{Components of the Toolkit}

\section{G1 - Introducing ABC learning design}

How can we engage and enable our time-pressured academics to design rich blended and online courses? Even before the Covid epidemic of early 2020, most leading universities already had ambitious strategies to develop digitally rich blended and fully online courses. 


\section{ABC LD - A new Toolkit for Rapid Learning Design}

Progress was patchy in most institutions, as only a few pioneering academics had the design skills, technology knowledge and above all time to remodel their programmes. Covid was, on top of everything else, a challenge to current learning designs and has forced even traditionally-minded universities to "pivot" rapidly to online learning provision, a cultural transformation that is unlikely to be completely reversed. Many universities with experience of $\mathrm{ABC} \mathrm{LD}$ are now looking to integrate the method into their response. The "Base" version of ABC LD is a high-energy hands-on workshop. In just 90 minutes teaching teams work together to create a visual "storyboard". The storyboard is made up of preprinted cards representing the type and sequence of learning activities (both online and offline) required to meet the module or programme learning outcomes. Assessment methods, cross-programme themes and institutional policies can all be integrated into the process. The key to this approach is pace, engagement and collaboration. The ABC LD method was found particularly useful for new programmes or those changing to an online or more blended format. The approach generates high levels of engagement, creative informed dialogue and group reflection about curriculum design among even time-poor academics. The workshops have an immediate impact in terms of stimulating a level of collaborative "educational design thinking" in a range of academic contexts. An early form of the Toolkit was released in 2018. G1 discusses the underlying pedagogy of ABC LD, namely Professor Diana Laurillard's concept of "learning types", itself derived from her "Conversational Framework" model of adult learning (Laurillard, 2002). This has proved a remarkably robust and accessible route into teaching and learning discussion and reflection. The six learning types are: Acquisition (i.e.to read/watch/listen), Investigation, Practice, Discussion, Collaboration and Production. For teachers with limited experience of (and sometimes enthusiasm for) educational theory, the learning types approach is easy to grasp, resonates with practice, and stimulates rich pedagogical conversation. The six learning types were converted into the $\mathrm{ABC}$ LD cards, (Figure 1) used to create the storyboard. 


\section{Learning types cards (front)}
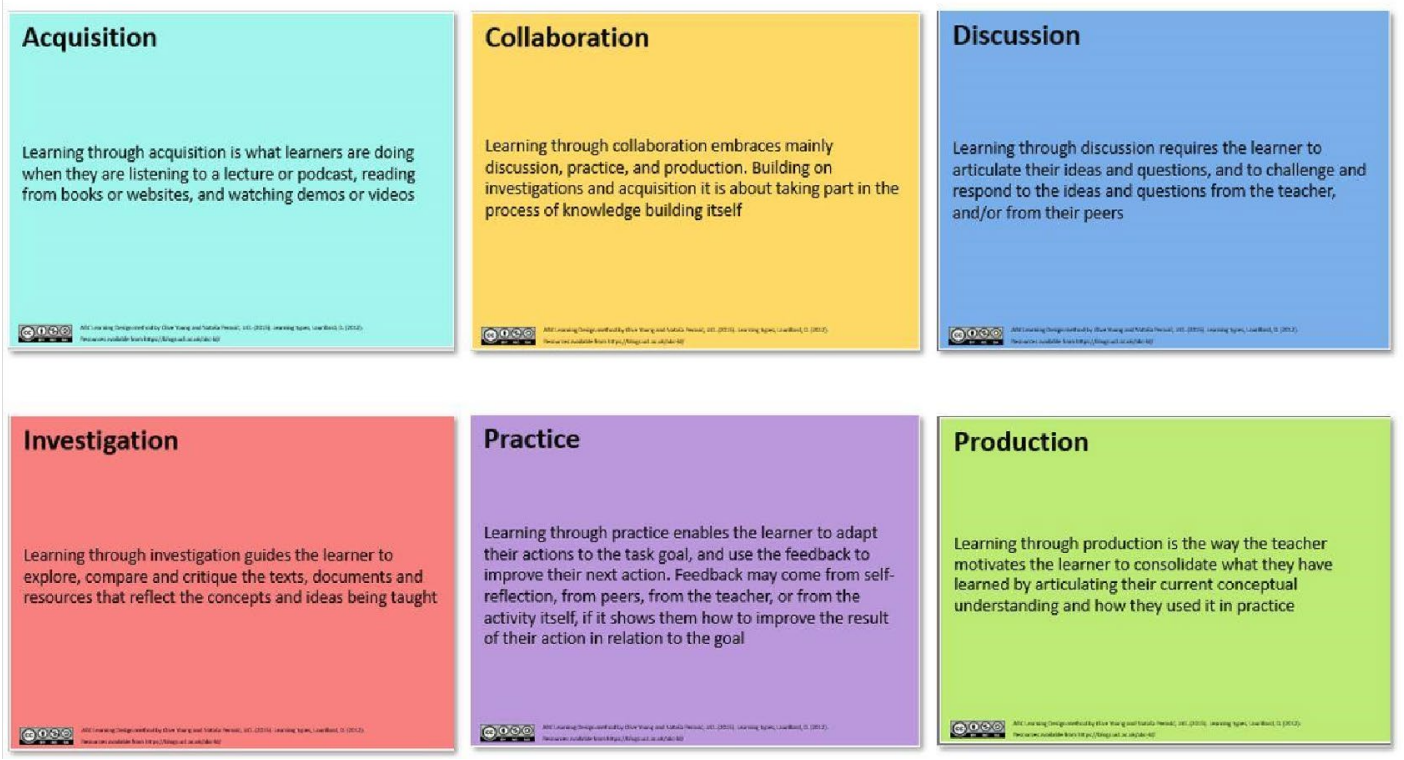

Figure 1. ABC LD Learning Types cards (front)

\section{G2 - Base ABC and translations}

This part of the toolkit explains how the Base (i.e. UCL) version of ABC LD workshop is structured, with detailed step-by-step advice on how to run it in that format, why the event is structured that way and potential questions the organisers (and participants) might have. The workshop is organised in a very planned and time-conscious manner (Figure 2). Most of the 90 minutes is spent on group activity, but it starts with a brief presentation introducing the toolkit elements and their pedagogical background.

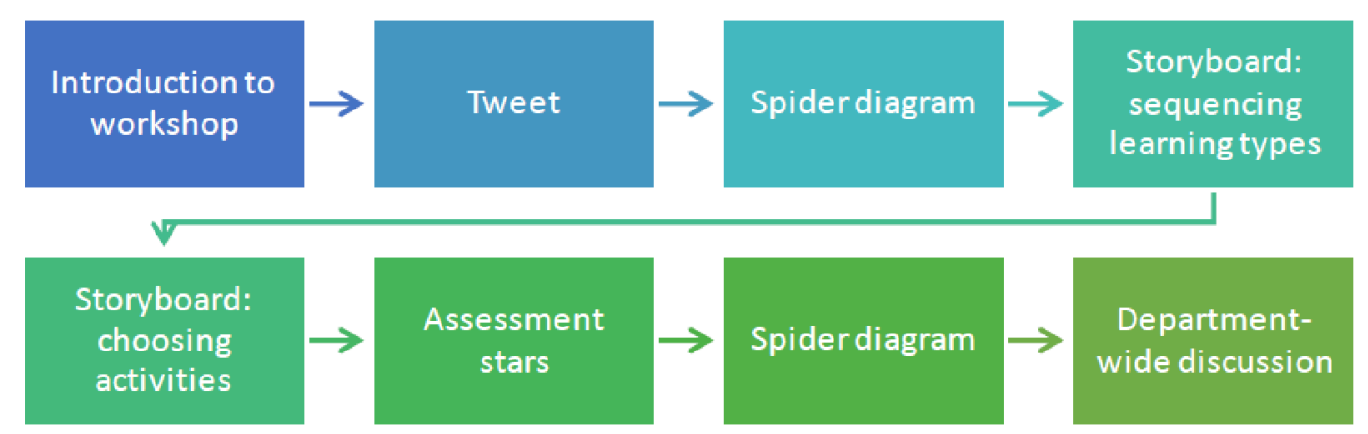

Figure 2. ABC workshop activities as a flow chart (Pieroni, 2019) 


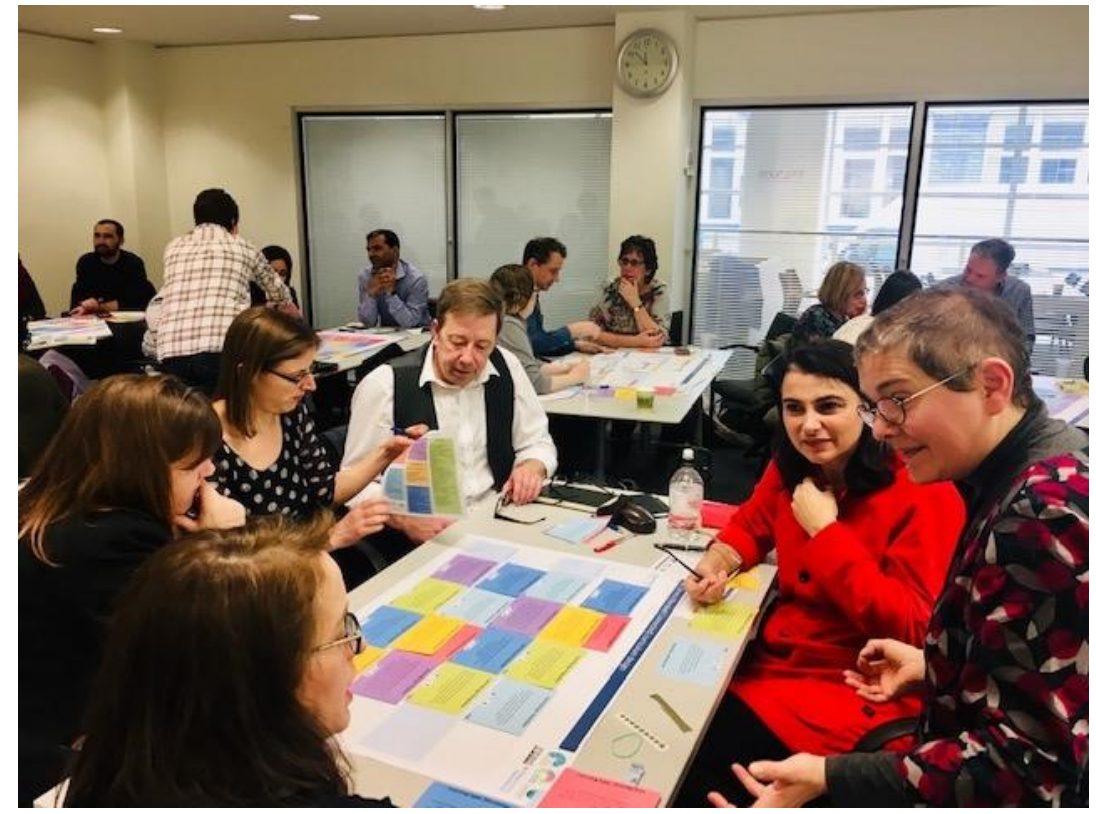

Figure 3. Facilitating the ABC LD workshop

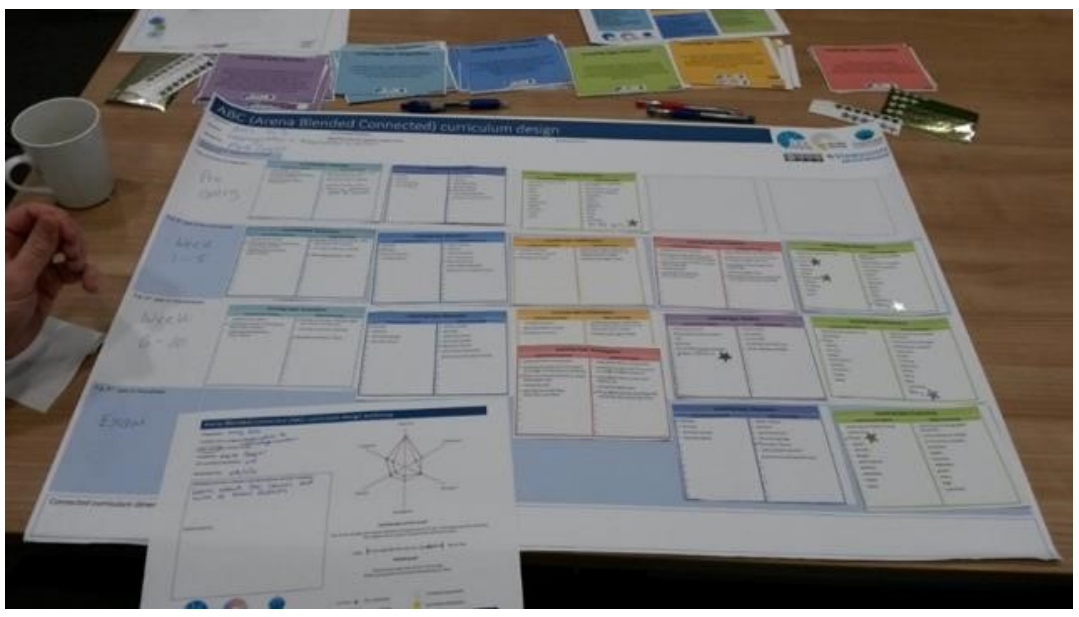

Figure 4. A completed ABC LD storyboard

The Toolkit includes ABC LD resources translated into 14 languages by members of the Erasmus+ project and the ABC LD community.

\section{G3 - Localising ABC}

While retaining the core principles, the "Base" workshop format is sometimes localised to specific institutional, teaching and discipline contexts. These variants are also described in the Toolkit. The examples of the local adaptations are given explaining why and how the changes have been made as well as the outcome of the changes. Apart from translation of the resources are translated, the timings may be changed (often extended), and the content, activities, card sets and storyboarding have all been adapted by different partners to fit with their institutional and national educational priorities. The ABC LD workshop is often supplemented by pre- and post-workshop events and activities and "extensions" included, such as mapping learning outcomes, competencies and so on. 
The ABC LD workshop can be supplemented by pre- and post-workshop events and activities and "extensions" included, such as mapping onto graduate attributes, assessment policy, learning analytics and other local priorities. It became clear during the project that although the UCL vision of ABC LD had focused on learning design, with some strategic components, the method was often applied in other areas, too. These areas can be summarised as follows:

- $\quad$ Learning Design - blended courses, programmes, MOOCs, CPD, training;

- Strategic Development - Research based learning, digital capabilities, employability, assessment and feedback review, student input;

- Academic Development - identification of skills, share practice, terminology, part of courses, practical development exercises, certification, case studies;

- Review of technical and support environment - VLE review, gap analysis, service provision, "app wheel”;

- Quality Assurance - documented part of new module/programme design and review;

- Analytics - identification of data points for "in-flight" feedback and post hoc review (with JISC, EUNIS);

- $\quad$ Digital capabilities for staff and students.

The Toolkit includes institutional narratives from the Erasmus+ partners.

\section{G4 - ABC LD and the VLE}

As colleagues from Dublin City University note, "It is clear that academic staff require further support to integrate technology within their teaching and specifically in their VLE and technology-enhanced practice". The hands-on team-based format of the classic ABC LD workshops is motivating and enjoyable and there is ample evidence of engagement and staff learning as a result. Early evaluation at UCL had shown however where there was a lack of direct follow-up support, participants were often unable fully realise the plans they made during workshops. Direct support by digital education technologists may be hard to scale so is invariably supplemented or replaced by online resources. Universities generally have extensive guidance supporting the tools in the VLE but the link to learning design is not always clear. Although not part of the Base workshop, the "Tool Wheel", where the tools available to teachers - in the VLE and beyond - are mapped to the learning types, has proved a critical part of institutional implementation (see Figure 5). 


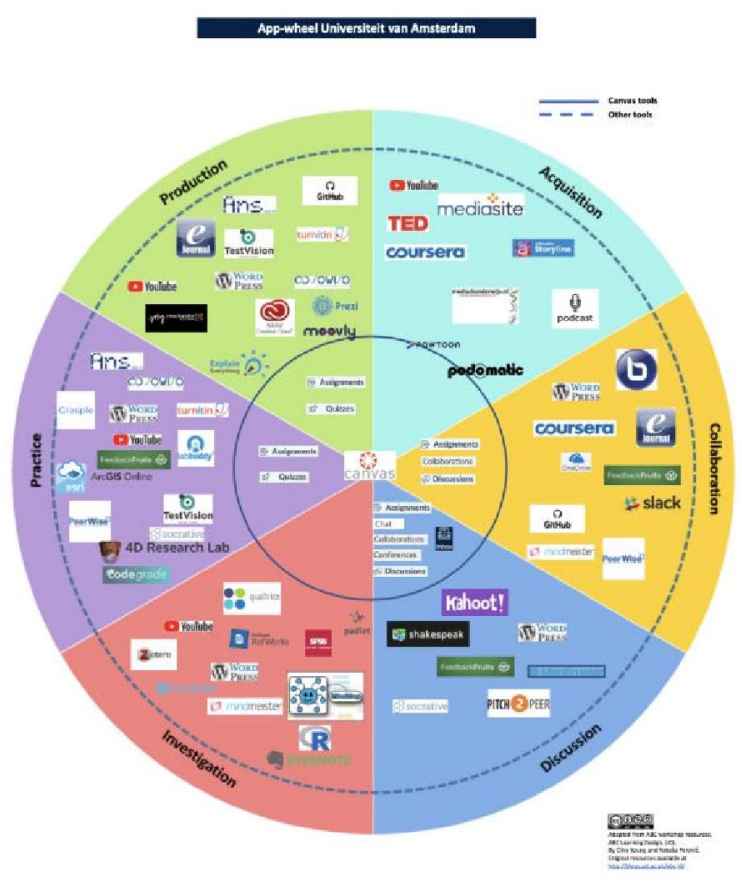

Figure 5. Example Tool Wheel from the University of Amsterdam

The Erasmus+ project explored several different implementations of the Tool Wheel concept. Four approaches were taken. Dublin City University produced an interactive online version using the web-based development environment HP5, Absalon developed a selection tool in WordPress, and UCL developed a worksheet. The deepest integration was from Vives, who had already been using ABC LD for several years when the project started. The Toolkit provides explanations of, and links to, each if these implementations. The H5P tool includes instructions how to localise the interactive tool to the institutional VLE and other supported tools.

\section{G5 - Does ABC LD work?}

At first, ABC LD can seem a challenging methodology. The dynamic, group based, rapid development format may feel quite different to existing methods of curriculum design, and even "normal" academic development events. The questions from people unfamiliar with the method are obvious. How will our academic colleagues react? Can such an apparently simple method achieve any useful results? Does it work for all disciplines? Won't sceptical and resistant colleagues disrupt the workshop? What if I can't answer the questions that come up? These, and others, are all perfectly valid concerns, and not easy to answer without experiencing the workshop directly. Since launching ABC LD in 2015, the UCL team have therefore made a considerable effort to facilitate "train the trainer" ABC LD workshops in many different institutions, and indeed that was a central component of the Erasmus+ project. The belief remains that by seeing how the format works by participating, attendees can judge for themselves whether it was relevant and useful for themselves and their institutions. In parallel, ABC LD has progressed through several 


\section{ABC LD - A new Toolkit for Rapid Learning Design}

stages of more structured evaluation, from initial ad hoc feedback through qualitative evaluation during a project funded by the Higher Education Funding Council England (HEFCE, 2018) to a more quantitative, survey-based approach as part of the Erasmus+ project (references). A few representative participant responses of the HEFCE research are as follows.

"We haven't had such level of detailed discussion as a team. I think the structure and the materials are facilitated well."

"It makes you think about: OK, we are going to use this technique, but where, how, for what and how does it fit with everything else? And this is the way into that, I think."

"It was an eye opener. I found it really useful to think about categorising how the learning objectives will be delivered and assessed, and examining the variety of ways that these can be achieved. It made me think more deeply about what skills the students can develop by making them responsible for their learning journey and not simply the content that needs to be delivered to them".

For the ABC to VLE project evaluation, a questionnaire was agreed by the partners who ran 84 ABC Learning Design workshops in 11 countries, with more than 1035 participants. Feedback was sought from over 60 of the workshops and the project team received 344 participant responses and a further 42 follow up responses about whether they had implemented their plans. The full report is available (https://abc-ld.org/evaluation/) but Figure 6 encapsulates the main message.

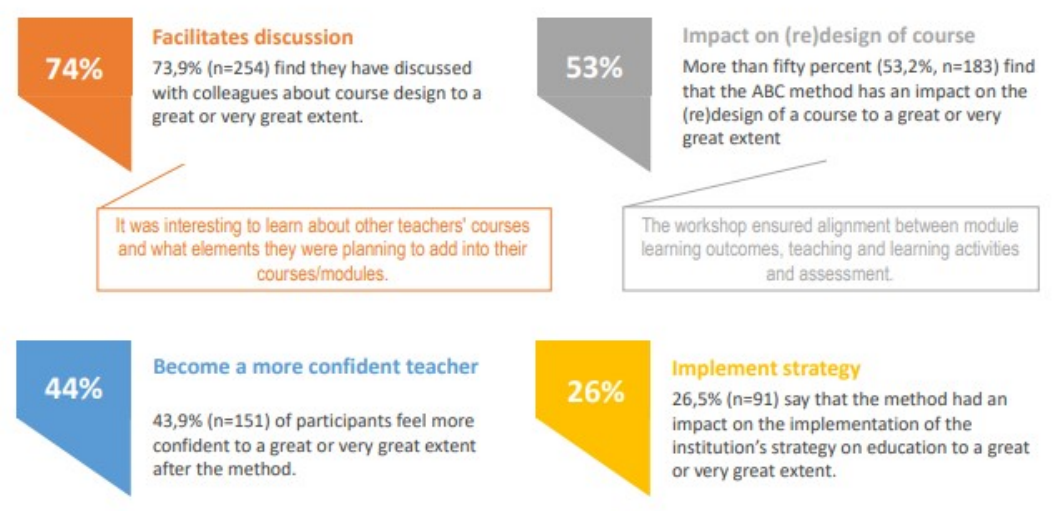

Figure 6. To what extent did you reach the following outcomes by the end of the workshop?

The opportunity, perhaps "permission" to discuss how to design the student journey in a non-judgmental, collegiate atmosphere is always much appreciated. Although only small majority found it had an immediate impact on course redesign, it should be remembered 
ABC LD is a short-form intervention with academic teams with little or no preparation. Moreover, "redesign" of the course in terms of changes may not be the only desirable outcome. The examination and overt justification of the current course may itself be valuable and this relates to the next point. The finding that participants feel more confident teachers after $A B C L D$ is as unexpected as it is pleasing. We have often seen that the openness of $\mathrm{ABC}$ LD can help validate current practices and designs among peers. Teachers can be quite self-critical of their own methods but may find, when discussing with colleagues, that the underlying rationale is quite robust and may require only minor adjustments. Thus, even if few changes are made to the design, the outcome can be satisfying. Not all workshops (at least prior to Covid) focus on implementing educational strategy or policy, so it was almost surprising that over a quarter of respondents recognised a strategic component.

\section{G6 - Online ABC LD (Covid versions)}

Due to social distancing demands, the community cannot run the popular face-to-face $\mathrm{ABC}$ LD workshops at the time of writing. However, many institutions wish to continue to use the method. The community has been very active in sharing their experiences. There are three components of $\mathrm{ABC} L \mathrm{LD}$ that are often repurposed for online delivery. The first is the concept of the learning types. As mentioned above, the learning types framework types has proved a rapid, robust and engaging route into pedagogical discussion and reflection. Even without the complete ABC LD workshop, learning types act as a focus for consideration of existing (face-to-face or blended) teaching and learning activities and what fully online alternatives might be. One example is to use a simple worksheet categorising the six types in terms of "conventional" and digital practices, how they might be implemented in the VLE and some ideas for engaging activities using these tools. The App Wheel as in Figure 5 provides another approach to linking pedagogy and technology, providing a clear visual representation of the toolset available and where it can be used. Although drawing from ABC LD, these are essentially staff development activities. The most challenging component of $\mathrm{ABC}$ LD to replicate online is also the one that participants usually find the most rewarding: the collaborative storyboarding. While there is no single tool or approach that meets this requirement, several approaches have been tried by the community. One is to use a shared Microsoft PowerPoint space, as the base version tools (primarily the cards and storyboard) are already in this format. The participants work synchronously in the shared online worksheet and "drag and drop" cards along the timeline. Popular shared "whiteboard" tools such as Padlet, Miro and Microsoft Planner have also been used to provide some of the dynamic interaction of the live workshop. Trello is a card-based online organiser that also shows some promise. UCL is experimenting with Learning Designer (https://www.ucl.ac.uk/learning-designer/9 a session planning tool from UCL aimed at secondary school teachers but based on the same learning types pedagogical model as ABC 
LD. While not designed for course-level planning, it produces a useful list of actions. Various members of the ABC LD community, including UCL, have produced Microsoft Excel sheets that can be used to track decisions in ABC LD workshop and produce an action list. It may be that no single software tool will emerge, but institutions will adopt a variety of tools and workflows according to local licensing availability, context and needs. Although the $\mathrm{ABC}$ to VLE project is now complete, the project partners continue to evaluate online alternatives and will add these to the Toolkit as they emerge (https://abcld.org/online-abc-ld/).

\section{G7 - The future of $A B C L D$}

During the final (online) meeting of the Erasmus+ project in June 2020, one partner remarked that the "Toolkit" was more than simply a set of downloadable guides, resources, tools and videos. Although we know of universities that have adopted ABC LD and have not contacted UCL or any other current user, the Toolkit artefacts themselves take on more value if they are placed in the context of shared professional practice. New users are recommended to discuss with UCL or other partners to form a better understanding of $\mathrm{ABC} L \mathrm{~L}$ and the ethos behind it. In the next stage of ABC LD, the partnership hopes to develop a structure of national information "hubs", local online communities, and an international conference to share ideas and resources. A method of "badging" facilitators to recognise their skills and experience has already been planned. Above all we anticipate that users of the Toolkit will wish to join the wider community, not only to gain ideas and updates, but to contribute to it themselves. The underpinning pedagogy of ABC LD is the "Conversational Framework" and the workshop in both live and online formats is also essentially a conversation. We hope the international ABC LD community will evolve into a global conversation, not only about $\mathrm{ABC}$ LD, which is just one method, but about the importance of learning design to build effective and engaging educational experiences.

\section{References}

ABC Learning Design. (2018). ABC to VLE partners. Retrieved from http://abcld.org/abc-to-vle/

ABC Learning Design. (2020). ABC LD Toolkit. Retrieved from https://abc-ld.org

Higher Education Funding Council for England (HEFCE). (2018).

https://www.gov.uk/government/organisations/higher-education-funding-councilfor-england

Laurillard, D. (2002). Rethinking University Teaching: A Conversational Framework for the Effective Use of Learning Technologies ( $2^{\text {nd }}$ ed.). London: RoutledgeFalmer. 
Laurillard, D. (2012). Teaching as a design science: Building pedagogical patterns for learning and technology. London: Routledge.

Pieroni, T. (2019). How do the experiences of those involved in course design impact the effective implementation of a blended curriculum, through the example of the ABC method? MA Thesis, MA Education and Technology, UCL Institute of Education.

Young, C. \& Perovic, N. (2016). Rapid and Creative Course Design: As Easy as ABC? Procedia - Social and Behavioral Sciences, 228, 390-395. 